\title{
PTS - Pavlovian Temperament Survey, versão adolescente/adulto: consistência interna e normatização para a realidade brasileira ${ }^{1}$
}

\author{
Raquel Souza Lobo Guzzo \\ Pontifícia Universidade Católica de Campinas \\ Ricardo Primi \\ Universidade São Francisco \\ Patrícia do Carmo Pereira Ito \\ Pontifícia Universidade Católica de Campinas
}

\begin{abstract}
Resumo
Este estudo objetivou verificar a consistência interna da Escala Pavlovian Temperament Survey (PTS), versão adolescente/adulto, descrever e estabelecer normas para avaliação do temperamento de adolescentes. A Escala PTS, versão adolescente/adulto, investiga três fatores de temperamento: Força de Excitação (FE), Força de Inibição (FI) e Mobilidade (MO). Participaram da pesquisa 952 adolescentes, de ambos os sexos, com idades entre 14 e 18 anos. Os índices de consistência interna obtidos apresentaram-se satisfatórios (FE = 0,71; FI = $0,69 ; \mathrm{MO}=0,74)$. Na caracterização do temperamento, os adolescentes apresentaram pontuações médias maiores nos fatores MO e FI e acentuadamente menores em FE. Diferenças significativas nas médias de MO e FE foram constatadas, quando considerado o sexo dos adolescentes. Os resultados obtidos asseguram a importância da PTS para avaliação do temperamento, sendo aconselhável a ampliação da amostra e delineamentos diferenciados em estudos futuros.
\end{abstract}

Palavras-chave: avaliação de temperamento; pavlovian temperament survey; técnicas psicométricas

\begin{abstract}
PTS - Pavlovian Temperament Survey, adolescent/adult version: internal consistency and normatization for Brazilian reality. This study aimed to verify the internal consistency of Pavlovian Temperament Survey (PTS) adolescent/ adult version, to describe and to establish norms for assessment of adolescents temperament. The PTS investigates three temperament factors: Strength of Excitation (SE), Strength of Inhibition (SI) and Mobility (MO). 952 adolescents participated in the research, from both gender, with ages between 14 and 18 years. The indexes of internal consistency obtained were considered satisfactory ( $\mathrm{SE}=0,71$; $\mathrm{SI}=0,69$; $\mathrm{MO}$ $=0,74)$. In the characterization of the temperament, the adolescents presented mean scores higher in the factors MO and SI, and strongly smaller in SE. Significant differences in the means of MO and SE were verified when sex was considered. The results assure the importance of PTS for assessment of temperament, and suggestions are presented regarding sample enlargement and design differentiation in future studies.
\end{abstract}

Key words: temperament assessment; pavlovian temperament survey; psychometrics techniques

$\mathrm{N}$ o campo de estudo da Psicologia, grande ênfase é atribuída ao estudo das diferenças individuais: variados autores, embasados em diferentes abordagens teóricas, tentam compreender e explicar a individualidade humana. Dentre as diversas características estudadas na comunidade científica internacional, principalmente Europa e Estados Unidos, pesquisas têm dedicado atenção especial ao temperamento, sua avaliação e aplicabilidade na área clínica e escolar (Guzzo, Pereira, \& Valli, submetido).
Pesquisado por diferentes autores, o temperamento tem sido estudado com diferentes fundamentações teóricas as quais vão influenciar na sua definição, dimensões constituintes e instrumentos de avaliação (Ito \& Guzzo, 2002). Para uma compreensão do que seja este construto, Goldsmith e Rieser-Danner (1986) apresentam alguns aspectos que são característicos do temperamento e se constituem em pontos comuns presentes nas diferentes concepções teóricas: (1) o temperamento está relacionado a dimensões gerais de comportamento, as quais caracterizam diferenças individuais 
representando padrões universais de desenvolvimento; (2) as características individuais temperamentais aparecem durante a infância e representam parte da fundamentação da personalidade; (3) as dimensões temperamentais são relativamente estáveis ao longo do tempo; (4) os traços de temperamento apresentam substrato biológico e (5) a expressão das características temperamentais podem sofrer influências de fatores do contexto.

Resumidamente, temperamento tem sido considerado como a característica mais representativa da personalidade humana (Chess \& Thomas, 1986; Goldsmith et al.,1987; Hofstee \& De Raad, 1992; Strelau, 1991), compreendido como um traço, ou seja, um padrão de comportamento que diferencia as pessoas entre si e as habilita a enfrentar as situações do cotidiano de forma característica e própria.

O estudo do temperamento tem origens remotas e baseiase em distintas teorias que procuram explicar suas principais dimensões e funcionamento. Tais teorias podem ser consideradas segundo três abordagens: (a) a psicopatológica, cujo principal interesse está na descrição de perfis temperamentais a partir de padrões de comportamento; (b) a psicogenética, com objetivo de descrição de estruturas e formas temperamentais de caráter genético, assim como aspectos pré-natais das futuras formas de comportamento e (c) a psicofisiológica, cuja prioridade está no funcionamento do sistema nervoso central e periférico, tempo e intensidade de reações, diferenciando os indivíduos em suas formas de se relacionar com os outros (Hoogendorp, 1992).

Pesquisas têm demonstrado a importância do temperamento para o desenvolvimento saudável. Segundo Teglasi (1995), a avaliação do temperamento constitui uma área ativa de pesquisa com aplicabilidade sobre uma variedade de aspectos do desenvolvimento e saúde mental, tais como formação de consciência, interação entre pares, problemas de comportamento, realização escolar, vulnerabilidade, resiliência e fatores de risco. A compreensão do temperamento como origem da individualidade permite ao profissional melhorar estratégias na educação e socialização, auxiliar na elaboração de programas de prevenção e intervenção (Teglasi, 1998) e orientar pais, professores e outros cuidadores no estabelecimento de relacionamentos mais responsivos e construtivos com a criança (Carey, 1989; Carey \& McDevitt, 1994; Chess \& Thomas, 1989).

Apesar da importância para o estudo do desenvolvimento humano e das várias pesquisas já realizadas, muitas controvérsias existem no campo de estudo do temperamento, em função das diferentes abordagens teóricas adotadas. O levantamento realizado por Strelau (1991) exemplifica claramente esta dificuldade: ele constatou a existência de mais de 80 traços destinados a descrever o temperamento de adultos, alguns com nomenclaturas similares avaliando características diferentes, outros com nomenclaturas diferentes avaliando o mesmo traço. A diversidade de concepções teóricas delimitando diferentes traços de temperamento induz os pesquisadores a utilizarem diferentes instrumentos para investigação das características temperamentais. A este respeito, Strelau (1998) cita que existem
21 instrumentos para avaliação do temperamento de crianças e 17 para avaliação do temperamento de adultos. Porém, muitos desses instrumentos não apresentam estudos sobre suas qualidades psicométricas, evidenciando a necessidade de pesquisas sobre a confiabilidade do instrumento de avaliação.

A importância da construção e da adaptação e validação de instrumentos de avaliação do temperamento nas diferentes culturas tem sido bastante ressaltada, pois proporcionam a possibilidade de realização de estudos transculturais, os quais possibilitam investigar a extensão em que as características temperamentais são universais e a maneira específica de expressão destas características em diferentes culturas (Strelau, Angleitner, \& Newberry, 1999).

No Brasil, o estudo do temperamento é escasso, mas vem sendo, aos poucos, estimulado, principalmente no âmbito da Psicologia Escolar, como forma de instrumentalizar o psicólogo na realização de avaliações das diferenças individuais de adolescentes em situação de escolha profissional e em outras questões relacionadas ao desenvolvimento da escolaridade e das atividades profissionais (Riello, 1992). As necessidades práticas, no entanto, esbarram com a dificuldade de se assegurar a forma de avaliação dessas características. Por essa razão, a construção de normas para a avaliação do temperamento de adolescentes brasileiros, a partir de um instrumento já adaptado para a realidade do país, torna-se especialmente importante (Guzzo, Riello, \& Primi, 1996). Além disso, a importância da padronização de medidas de atributos psicológicos atende a critérios de clarificação da presença dessa característica na população. Como afirma Nunnaly (1967), uma razão para se enfatizar a necessidade de estudos e medidas de um atributo em particular exige a cautelosa definição da natureza do que vai ser medido, antes de fazê-lo, porque, uma das possibilidades nesse caso, é a de que esse atributo não exista na população ou apresente-se de forma conjunta com outros elementos a serem medidos.

Esta conclusão somente poderá ser aceita se forem obtidos resultados negativos em estudos de descrição e avaliação desses atributos em diferentes populações. Por esta razão, as pesquisas envolvendo a construção, adaptação ou validação de instrumentos psicológicos estão longe de se concluir e seus resultados se apresentarem como definitivos para uma determinada população. Há sempre que se atualizar o instrumento com verificações periódicas de sua validade ou precisão para a população estudada (Pasquali, 1997).

A medida é uma importante característica de um campo de estudo, pois aperfeiçoa a distinção que se pode fazer de um fenômeno em relação a outros, ao mesmo tempo em que assegura a habilidade em descrever o que ocorre na área (Krathwohl, 1993). Por essa razão, os estudos sobre a avaliação do temperamento, na realidade brasileira, devem continuar de forma a propiciar o aprimoramento das características psicométricas de suas medidas.

Considerando a importância dos estudos sobre temperamento para o desenvolvimento do indivíduo, a carência de instrumentos de avaliação das características de temperamento no Brasil e a importância da existência de testes 
validados, precisos e normatizados para a realidade brasileira, este estudo objetiva: (1) estabelecer validade interna e precisão da escala Pavlovian Temperament Survey - PTS, versão adolescente/adulto, para a avaliação do temperamento; (2) descrever o perfil temperamental de adolescentes de ambos os sexos com idade de 14 a 18 anos, provenientes de diferentes tipos de escolas e compará-lo segundo as variáveis independentes - sexo e idade; e (3) estabelecer normas para a avaliação do temperamento de adolescentes segundo a escala Pavlovian Temperament Survey.

Tabela 1

Distribuição geral da freqüência e porcentagem das variáveis demográficas

\begin{tabular}{llcc}
\hline & Variáveis & Freqüência & $\%$ \\
\hline Sexo & Masculino & 401 & 42,1 \\
& Feminino & 551 & 57,9 \\
Tipo de & Pública & 346 & 36,4 \\
& & & \\
& Particular & 600 & 63,0 \\
Idades & Sem & 6 & 0,6 \\
& Identificação & & \\
& 14 anos & 169 & 17,8 \\
& 15 anos & 223 & 23,4 \\
& 16 anos & 232 & 24,4 \\
& 17 anos & 173 & 18,2 \\
& 18 anos & 155 & 16,3 \\
\hline
\end{tabular}

\section{Método}

\section{Participantes}

Participaram desta pesquisa 952 adolescentes de ambos os sexos, com idades entre 14 e 18 anos, freqüentando diferentes séries do ensino fundamental e médio de escolas públicas e particulares da região de Campinas (Tabela 1). A amostra foi obtida pela técnica da estratificação (Krathwohl, 1993).

\section{Instrumento}

Para a coleta de dados foi utilizada a escala Pavlovian Temperament Survey (PTS), versão adolescente/adulto, desenvolvida na década de 1990 por Strelau, Angleitner, Batelman e Ruch (1999) e adaptada para o Brasil por Guzzo et al. (1996). Trata-se de uma escala construída com o objetivo de avaliar a expressão comportamental das propriedades do Sistema Nervoso Central, conforme entendido por Pavlov, de modo a permitir estudos transculturais. Em seu estudo brasileiro, a escala, do seu formato original de 252 itens, passou a conter 57 itens (Guzzo et al., 1996), versão utilizada no presente estudo.

Tal como proposta por seus autores originais, a PTS avalia três fatores de temperamento baseados nas propriedades pavlovianas de sistema nervoso: (1) Força de Excitação (FE), a qual se caracteriza por avaliar como o indivíduo reage frente à estimulação intensa e prolongada; (2) Força de Inibição (FI), que avalia a capacidade do indivíduo refrear um dado comportamento quando necessário e (3) Mobilidade (MO), que avalia a habilidade do indivíduo responder adequadamente a contínuas mudanças no ambiente. Cada fator é constituído por 19 itens (Tabela 2).

Os 57 itens da PTS são respondidos em conformidade com escala de formato Likert, com quatro possibilidades de resposta (concordo plenamente, concordo, discordo e discordo plenamente) e pontuação variando de 1 a 4 pontos.

A PTS é composta por um caderno que contém as instruções e os 57 itens, além de uma folha de resposta, que apresenta campos específicos para identificação e

Tabela 2

Distribuição dos itens da Pavlovian Temperament Survey (PTS), versão 14 a 18 anos, considerando as dimensões

\begin{tabular}{|c|c|}
\hline Dimensões & Itens \\
\hline Mobilidade (MO) $n=19$ & $3,6,9,12,15,18,21,24,27,30,33,36,39,42,45,48,51,54,57$ \\
\hline Força de excitação (FE) $n=19$ & $2,5,8,11,14,17,20,23,26,29,32,35,38,41,44,47,50,53,56$ \\
\hline Força de inibição (FI) $n=19$ & $1,4,7,10,13,16,19,22,25,28,31,34,37,40,43,46,49,52,55$ \\
\hline
\end{tabular}


caracterização dos participantes e campos específicos para assinalar a resposta a cada um dos itens da escala.

\section{Procedimento}

Mediante contato prévio com as instituições de ensino participantes da coleta de dados, foram realizadas entrevistas com as direções/coordenações, ocasião em que eram explicitados os objetivos e o caráter sigiloso da pesquisa, sendo agendada uma data e horário para aplicação da escala em sala de aula. Em sala de aula, os alunos eram convidados a participar da pesquisa, recebendo também informações sobre o objetivo, sigilo dos dados e a liberdade em optar por participar ou não da coleta de dados.

Feitos os esclarecimentos, os participantes recebiam o caderno contendo as instruções, os itens da escala e a folha de resposta. Em seguida era iniciada a aplicação da PTS com a leitura das instruções em voz alta e o esclarecimento de dúvidas. Após as instruções coletivas, os alunos eram orientados a começarem a ler e responder individualmente os itens da escala. A aplicação da PTS foi coletiva e dois pesquisadores permaneciam na sala para dar as orientações necessárias, esclarecer as dúvidas, distribuir e recolher o material.

Finalizada a fase de aplicação, os dados coletados foram inseridos e analisados no programa Statistical Package for the Social Science - SPSS for Windows (versão 10.0).

\section{Resultados}

Antes da apresentação dos resultados referentes à descrição do perfil temperamental e o estabelecimento de normas para avaliação do temperamento de adolescentes, é importante apresentar os dados referentes à consistência interna da escala PTS, versão adolescente/ adulto. Resultados obtidos por meio do cálculo do coeficiente alpha de Cronbach, nos três fatores mensurados pela escala, evidenciaram os seguintes índices de consistência interna: FI = 0,69; FE = 0,71 e $\mathrm{MO}=0,74$.

Definida a consistência interna, serão apresentados a seguir os resultados referentes à descrição do perfil de temperamento, considerando-se as variáveis independentes sexo e idade. O primeiro passo para a análise desses resultados consistiu na somatória dos pontos brutos obtidos pelos participantes em cada um dos fatores, sendo em seguida calculada a média e o desvio-padrão.

Considerando o grupo total de adolescentes participantes (Tabela 3), foi possível notar que o fator que obteve média mais elevada foi MO $(50,63)$, aparecendo em seguida FI $(50,13)$ com pontuação média aproximada a MO e, por último, com uma média mais baixa, FE $(41,46)$.

No que diz respeito às médias obtidas nos fatores, quando considerada a variável independente sexo, observou-se que os adolescentes do sexo masculino apresentaram médias mais elevadas em MO e FE, quando comparados aos do sexo feminino. Em contrapartida, adolescentes do sexo feminino apresentaram pontuação média um pouco maior em FI (Tabela 3). A distribuição das médias nos fatores avaliados pela PTS (Tabela 3), evidenciou que adolescentes do sexo masculino apresentaram o mesmo padrão de distribuição da amostra total de participantes, ou seja, MO com média mais elevada, seguida pela média intermediária em FI e, por último, pela menor média em FE ( $\mathrm{MO}>\mathrm{FI}>\mathrm{FE})$. As adolescentes femininas apresentaram uma distribuição um pouco diferenciada: FI com média maior do que $\mathrm{MO}$ e, por último, permanecendo $\mathrm{FE}$ (FI > $\mathrm{MO}>\mathrm{FE}$ ).

Quando considerada a variável independente idade (Tabela 4), demonstraram que os adolescentes de 16 anos obtiveram médias mais elevadas nos fatores MO e FI, quando comparados aos participantes de outras idades. No que diz

Tabela 3

Média e desvio-padrão (dp) obtidos nas dimensões da PTS, versão 14 a 18 anos, considerando-se o grupo total de participantes e a variável sexo

\begin{tabular}{|c|c|c|c|c|c|c|}
\hline \multirow{3}{*}{ Fatores } & \multirow{2}{*}{\multicolumn{2}{|c|}{$\begin{array}{l}\text { Total sujeitos } \\
\quad(N=952)\end{array}$}} & \multicolumn{4}{|c|}{ Sexo } \\
\hline & & & \multirow{2}{*}{$\begin{array}{c}\text { Masculino } \\
\text { Média }\end{array}$} & \multicolumn{2}{|c|}{$n=401)$ Feminino (} & \multirow{2}{*}{$\begin{array}{c}n=551) \\
d p\end{array}$} \\
\hline & Média & $d p$ & & $d p$ & Média & \\
\hline Mobilidade & 50,63 & 6,86 & 51,58 & 7,15 & 49,49 & 6,31 \\
\hline $\begin{array}{l}\text { Força de } \\
\text { excitação }\end{array}$ & 41,46 & 7,13 & 42,47 & 7,22 & 40,73 & 6,97 \\
\hline $\begin{array}{l}\text { Força de } \\
\text { inibição }\end{array}$ & 50,13 & 6,69 & 49,98 & 6,93 & 50,23 & 6,52 \\
\hline
\end{tabular}


Tabela 4

Média e desvio-padrão (dp) obtidos nas dimensões da PTS, versão 14 a 18 anos, considerando-se a variável idade

\begin{tabular}{lcccccccccc}
\hline \multirow{2}{*}{ Fatores } & \multicolumn{10}{c}{ Idade } \\
\cline { 2 - 11 } & \multicolumn{1}{c}{14 anos } & \multicolumn{1}{c}{15 anos } & \multicolumn{1}{c}{16 anos } & 17 anos & 18 anos \\
\cline { 2 - 11 } & Média & $d p$ & Média & $d p$ & Média & $d p$ & Média & $d p$ & Média & $d p$ \\
\hline Mobilidade & 50,18 & 6,62 & 50,43 & 6,82 & 51,36 & 7,18 & 50,26 & 6,69 & 50,74 & 6,85 \\
Força de excitação & 42,11 & 7,23 & 41,65 & 7,46 & 41,79 & 7,29 & 41,12 & 6,88 & 40,37 & 6,48 \\
Força de inibição & 48,73 & 6,10 & 49,68 & 6,84 & 50,91 & 6,46 & 50,49 & 6,73 & 50,73 & 7,18 \\
& & & & & & & & & & \\
\hline
\end{tabular}

respeito ao fator $\mathrm{FE}$, este obteve maior média entre os adolescentes de 14 anos.

Quanto à distribuição das médias, constatou-se que esta se manteve constante entre os adolescentes de diferentes idades, MO foi a dimensão com média mais elevada, seguida por FI, com média ligeiramente mais baixa e, por último, FE com média acentuadamente menor ( $\mathrm{MO}>\mathrm{FI}>\mathrm{FE})$. A exceção a esta distribuição ficou por conta dos adolescentes de 17 anos, para os quais FI apresentou média mais elevada do que MO, porém FE permaneceu com menor média (FI > MO > FE).

Para verificar a variância das médias obtidas nos fatores mensurados pela PTS, foi realizada a ANOVA de medidas repetidas ( 2 x 5 x 3), ou seja, envolvendo dois sexos (masculino e feminino), cinco idades (14, 15, 16, 17 e 18 anos) e três fatores da PTS (FE, FI e MO). Os resultados evidenciaram diferenças significativas entre as médias nos fatores mensurados pela escala $\left(F_{(2,9)}=684,5 ; p<0,001\right)$, interações significativas entre os fatores da PTS e a variável sexo $\left(F_{(2,9)}\right.$ $=4,1 ; p=0,016)$ e entre os fatores da PTS e a variável idade $\left(F_{(8,19)}=2,3 ; p=0,019\right)$.

A seguir serão apresentados os resultados referentes à normatização, a qual foi estabelecida a partir do cálculo do percentil acumulado para os valores brutos possíveis de serem obtidos em cada um dos três fatores (FE, FI e MO) avaliados pela PTS. É importante, neste ponto, ressaltar que cada um dos fatores é constituído por 19 itens e cada item pode receber uma pontuação que varia de 1 a 4 pontos. Portanto, a pontuação bruta em cada um dos fatores pode variar entre o mínimo de 19 pontos e o máximo de 76 pontos.

Considerando o grupo total de adolescentes (Tabela 5), é possível observar que o fator $\mathrm{FE}$ foi o que apresentou maior porcentagem de adolescentes (18\%) com a pontuação mínima, enquanto que nos fatores FI e MO a porcentagem de participantes com pontuação mínima foi consideravelmente menor, respectivamente $3 \%$ e $4 \%$. FE também foi o fator que apresentou maior percentil acumulado de adolescentes com a pontuação máxima (98\%).
Tabela 5

Percentil acumulado para o grupo total de adolescentes de 14 a 18 anos $(N=952)$

\begin{tabular}{|c|c|c|c|c|c|c|c|c|c|c|c|}
\hline \multirow{2}{*}{$\begin{array}{l}\text { Resultado } \\
\text { bruto }\end{array}$} & \multicolumn{3}{|c|}{ Percentis } & \multirow{2}{*}{$\begin{array}{l}\text { Resultado } \\
\text { bruto }\end{array}$} & \multicolumn{3}{|c|}{ Percentis } & \multirow{2}{*}{$\begin{array}{l}\text { Resultado } \\
\text { bruto }\end{array}$} & \multicolumn{3}{|c|}{ Percentis } \\
\hline & $\mathrm{FE}$ & FI & $\mathrm{MO}$ & & $\mathrm{FE}$ & FI & MO & & FE & FI & MO \\
\hline 19 & 18 & 3 & 4 & 39 & 47 & 33 & 33 & 59 & 75 & 63 & 62 \\
\hline 20 & 20 & 5 & 5 & 40 & 48 & 35 & 34 & 60 & 76 & 65 & 64 \\
\hline 21 & 21 & 6 & 7 & 41 & 49 & 36 & 36 & 61 & 77 & 66 & 65 \\
\hline 22 & 23 & 8 & 8 & 42 & 51 & 38 & 37 & 62 & 79 & 68 & 67 \\
\hline 23 & 24 & 9 & 10 & 43 & 52 & 39 & 39 & 63 & 80 & 69 & 68 \\
\hline 24 & 25 & 11 & 11 & 44 & 54 & 41 & 40 & 64 & 82 & 71 & 70 \\
\hline 25 & 27 & 12 & 13 & 45 & 55 & 42 & 42 & 65 & 83 & 72 & 71 \\
\hline 26 & 28 & 14 & 14 & 46 & 56 & 44 & 43 & 66 & 84 & 74 & 72 \\
\hline 27 & 30 & 15 & 16 & 47 & 58 & 45 & 45 & 67 & 86 & 75 & 74 \\
\hline 28 & 31 & 17 & 17 & 48 & 59 & 47 & 46 & 68 & 87 & 77 & 75 \\
\hline 29 & 33 & 18 & 18 & 49 & 61 & 48 & 48 & 69 & 89 & 78 & 77 \\
\hline 30 & 34 & 20 & 20 & 50 & 62 & 50 & 49 & 70 & 90 & 80 & 78 \\
\hline 31 & 35 & 21 & 21 & 51 & 63 & 51 & 51 & 71 & 91 & 81 & 80 \\
\hline 32 & 37 & 23 & 23 & 52 & 65 & 53 & 52 & 72 & 93 & 83 & 81 \\
\hline 33 & 38 & 24 & 24 & 53 & 66 & 54 & 53 & 73 & 94 & 84 & 83 \\
\hline 34 & 40 & 26 & 26 & 54 & 68 & 56 & 55 & 74 & 96 & 86 & 84 \\
\hline 35 & 41 & 27 & 27 & 55 & 69 & 57 & 56 & 75 & 97 & 87 & 86 \\
\hline 36 & 42 & 29 & 29 & 56 & 70 & 59 & 58 & 76 & 98 & 89 & 87 \\
\hline 37 & 44 & 30 & 30 & 57 & 72 & 60 & 59 & & & & \\
\hline 38 & 45 & 32 & 32 & 58 & 73 & 62 & 61 & & & & \\
\hline
\end{tabular}

Nota. FE $=$ Força de excitação $(m=41,46 ; d p=7,12) ;$ FI $=$ Força de inibição $(m=50,12 ; d p=6,69)$; $\mathrm{MO}=$ Mobilidade $(m=50,63 ; d p=6,85)$ 
Tabela 6

Percentil acumulado para adolescentes do sexo feminino de 14 a $18 \operatorname{anos}(\mathrm{n}=551)$

\begin{tabular}{|c|c|c|c|c|c|c|c|c|c|c|c|}
\hline \multirow{2}{*}{$\begin{array}{l}\text { Resultado } \\
\text { Bruto }\end{array}$} & \multicolumn{3}{|c|}{ Percentis } & \multirow{2}{*}{$\begin{array}{l}\text { Resultado } \\
\text { Bruto }\end{array}$} & \multicolumn{3}{|c|}{ Percentis } & \multirow{2}{*}{$\begin{array}{l}\text { Resultado } \\
\text { Bruto }\end{array}$} & \multicolumn{3}{|c|}{ Percentis } \\
\hline & $\mathrm{FE}$ & FI & MO & & $\mathrm{FE}$ & FI & MO & & $\mathrm{FE}$ & FI & MO \\
\hline 19 & 19 & 2 & 3 & 39 & 48 & 33 & 33 & 59 & 76 & 63 & 63 \\
\hline 20 & 20 & 4 & 5 & 40 & 49 & 34 & 35 & 60 & 78 & 65 & 65 \\
\hline 21 & 22 & 5 & 6 & 41 & 50 & 36 & 36 & 61 & 79 & 67 & 66 \\
\hline 22 & 23 & 7 & 8 & 42 & 52 & 37 & 38 & 62 & 81 & 68 & 68 \\
\hline 23 & 25 & 8 & 9 & 43 & 53 & 39 & 39 & 63 & 82 & 70 & 69 \\
\hline 24 & 26 & 10 & 11 & 44 & 55 & 40 & 41 & 64 & 83 & 71 & 71 \\
\hline 25 & 27 & 11 & 12 & 45 & 56 & 42 & 42 & 65 & 85 & 73 & 72 \\
\hline 26 & 29 & 13 & 14 & 46 & 58 & 44 & 44 & 66 & 86 & 74 & 74 \\
\hline 27 & 30 & 14 & 15 & 47 & 59 & 45 & 45 & 67 & 88 & 76 & 75 \\
\hline 28 & 32 & 16 & 17 & 48 & 60 & 47 & 47 & 68 & 89 & 77 & 77 \\
\hline 29 & 33 & 17 & 18 & 49 & 62 & 48 & 48 & 69 & 91 & 79 & 78 \\
\hline 30 & 35 & 19 & 20 & 50 & 63 & 50 & 50 & 70 & 92 & 80 & 80 \\
\hline 31 & 36 & 21 & 21 & 51 & 65 & 51 & 51 & 71 & 93 & 82 & 81 \\
\hline 32 & 37 & 22 & 23 & 52 & 66 & 53 & 53 & 72 & 95 & 83 & 83 \\
\hline 33 & 39 & 24 & 24 & 53 & 68 & 54 & 54 & 73 & 96 & 85 & 84 \\
\hline 34 & 40 & 25 & 26 & 54 & 69 & 56 & 56 & 74 & 98 & 86 & 86 \\
\hline 35 & 42 & 27 & 27 & 55 & 70 & 57 & 57 & 75 & 99 & 88 & 87 \\
\hline 36 & 43 & 28 & 29 & 56 & 72 & 59 & 59 & 76 & 100 & 90 & 89 \\
\hline 37 & 45 & 30 & 30 & 57 & 73 & 60 & 60 & & & & \\
\hline 38 & 46 & 31 & 32 & 58 & 75 & 62 & 62 & & & & \\
\hline
\end{tabular}

Nota. FE = Força de excitação $(m=40,73 ; d p=6,97) ;$ FI = Força de inibição $(m=50,23$; $d p=6,52)$; $\mathrm{MO}=$ Mobilidade $(m=50,25 ; d p=$ $6,66)$

Quando considerados somente adolescentes do sexo feminino (Tabela 6), FE também foi o fator que apresentou maior porcentagem de participantes com a pontuação mínima (19\%), aparecendo em seguida, com uma porcentagem bem menor, o fator MO (3\%) e FI (2\%). Como no grupo total, também foi $\mathrm{FE}$ o fator que apresentou maior percentil acumulado de participantes com a pontuação máxima.

Entre os participantes do sexo masculino (Tabela 7), tal como nas situações anteriores, FE foi o fator que apresentou a maior porcentagem de participantes com a pontuação mínima (18\%) e o maior percentil acumulado (96\%), quando comparado a FI e MO, que obtiveram uma porcentagem de 5\% de participantes com pontuação mínima e percentil acumulado de $88 \%$ em FI e $85 \%$ em MO.
Tabela 7

Percentil acumulado para adolescentes do sexo masculino de 14 a $18 \operatorname{anos}(\mathrm{n}=401)$

\begin{tabular}{|c|c|c|c|c|c|c|c|c|c|c|c|}
\hline \multirow{2}{*}{$\begin{array}{l}\text { Resultado } \\
\text { Bruto }\end{array}$} & \multicolumn{3}{|c|}{ Percentis } & \multicolumn{4}{|c|}{ Resultado Percentis } & \multicolumn{4}{|c|}{ Resultado Percentis } \\
\hline & $\mathrm{FE}$ & FI & MO & Bruto & $\mathrm{FE}$ & FI & MO & Bruto & FE & FI & MO \\
\hline 19 & 18 & 5 & 5 & 39 & 45 & 34 & 33 & 59 & 73 & 63 & 61 \\
\hline 20 & 19 & 7 & 6 & 40 & 47 & 36 & 34 & 60 & 74 & 64 & 63 \\
\hline 21 & 20 & 8 & 7 & 41 & 48 & 37 & 36 & 61 & 76 & 66 & 64 \\
\hline 22 & 22 & 10 & 9 & 42 & 49 & 38 & 37 & 62 & 77 & 67 & 65 \\
\hline 23 & 23 & 11 & 10 & 43 & 51 & 40 & 38 & 63 & 78 & 69 & 67 \\
\hline 24 & 24 & 12 & 12 & 44 & 52 & 41 & 40 & 64 & 80 & 70 & 68 \\
\hline 25 & 26 & 14 & 13 & 45 & 54 & 43 & 41 & 65 & 81 & 72 & 70 \\
\hline 26 & 27 & 15 & 14 & 46 & 55 & 44 & 43 & 66 & 83 & 73 & 71 \\
\hline 27 & 29 & 17 & 16 & 47 & 56 & 46 & 44 & 67 & 84 & 75 & 72 \\
\hline 28 & 30 & 18 & 17 & 48 & 58 & 47 & 46 & 68 & 85 & 76 & 74 \\
\hline 29 & 31 & 20 & 19 & 49 & 59 & 49 & 47 & 69 & 87 & 77 & 75 \\
\hline 30 & 33 & 21 & 20 & 50 & 60 & 50 & 48 & 70 & 88 & 79 & 77 \\
\hline 31 & 34 & 23 & 22 & 51 & 62 & 51 & 50 & 71 & 89 & 80 & 78 \\
\hline 32 & 36 & 24 & 23 & 52 & 63 & 53 & 51 & 72 & 91 & 82 & 79 \\
\hline 33 & 37 & 25 & 24 & 53 & 65 & 54 & 53 & 73 & 92 & 83 & 81 \\
\hline 34 & 38 & 27 & 26 & 54 & 66 & 56 & 54 & 74 & 94 & 85 & 82 \\
\hline 35 & 40 & 28 & 27 & 55 & 67 & 57 & 55 & 75 & 95 & 86 & 84 \\
\hline 36 & 41 & 30 & 29 & 56 & 69 & 59 & 56 & 76 & 96 & 88 & 85 \\
\hline 37 & 42 & 31 & 30 & 57 & 70 & 60 & 58 & & & & \\
\hline 38 & -44 & 33 & 34 & 58 & -71 & 62 & 60 & & & & \\
\hline
\end{tabular}

Nota. FE $=$ Força de excitação $(m=42,47 ; d p=7,22) ; \mathrm{FI}=$ Força de inibição ( $m=49,98 ; d p=6,92)$; $\mathrm{MO}=$ Mobilidade $(m=51,15 ; d p=$ $7,07)$

\section{Discussão}

Os resultados obtidos no presente estudo serão comparados aos dados obtidos por Strelau et al. (1999) em seus estudos transculturais, envolvendo a adaptação e utilização da PTS como instrumento de avaliação do temperamento em 16 países (Alemanha, Austrália, Bélgica, Brasil, Bulgária, Espanha, Estados Unidos, Coréia, Grã Bretanha, Grécia, Holanda, Hungria, Itália, Japão, Polônia, Romênia e Rússia) e aos resultados de estudos brasileiros realizados por Guzzo et al. (1996), objetivando investigar a consistência interna, clareza dos construtos FE, FI e MO e redução do número de itens da PTS, e por Riello (1999), que investigou o temperamento de adolescentes com diferentes envolvimentos em natação. 
A Escala PTS (Pavlovian Temperament Survey) apresentou neste estudo índices de consistência interna, considerados por Aron e Aron (1999), estatisticamente satisfatórios nas dimensões FE $(0,71)$ e MO $(0,74)$ e adequado em FI $(0,69)$.

A comparação destes dados com os índices de consistência interna obtidos por Guzzo et al. (1996), em uma outra amostra brasileira ( $\mathrm{FI}=0,73 ; \mathrm{FE}=0,80 ; \mathrm{MO}=0,83$ ), com os resultados obtidos por Strelau et al. (1999), na versão alemã da PTS, da qual foi adaptada a versão brasileira, (FI = 0,81; FE $=0,84 ; \mathrm{MO}=0,89$ ) e com a média dos índices obtidos nos estudos transculturais da escala $(\mathrm{FI}=0,79 ; \mathrm{FE}=0,84 ; \mathrm{MO}=$ 0,87 ) revelou que os índices obtidos neste estudo foram menores.

Diferenças nos índices de consistência interna entre os países são esperadas, pois a adaptação da PTS a diferentes culturas e linguagens envolve a tradução dos 252 itens que, originalmente, a constituem e a seleção dos itens mais representativos. Este processo de adaptação e seleção de itens faz com que as versões da PTS nos diferentes países passem a ser constituídas por diferentes itens em sentido e quantidade, fato que, segundo Strelau et al. (1999), pode influenciar no índice de consistência interna da escala.

É importante considerar também que este estudo envolveu a participação de adolescentes de 14 a 18 anos, enquanto que nos trabalhos de Guzzo et al. (1996) e Strelau et al. (1999) as amostras eram mais heterogêneas, constituídas por participantes com idades entre 10 e 85 . Talvez, a baixa precisão obtida entre os adolescentes pode ser indicador de uma maior dificuldade em compreender alguns dos itens do instrumento. No entanto, são necessários novos estudos para confirmar e investigar melhor a precisão do instrumento junto a amostras constituídas principalmente por adolescentes.

A respeito da variação dos índices de precisão envolvendo um mesmo instrumento em diferentes estudos, Anastasi (1988) ressalta que os coeficientes de precisão são dependentes e limitados à amostra na qual o teste foi aplicado e a partir da qual o coeficiente foi gerado.

No tocante às características de temperamento avaliadas pela PTS, os resultados obtidos indicaram que os adolescentes apresentaram como características marcantes de temperamento a Mobilidade (MO), que se refere a habilidade do indivíduo responder tão adequadamente quanto necessário a contínuas mudanças no ambiente e a Força de Inibição, que se refere a capacidade do indivíduo de interromper um determinado comportamento quando necessário, ou efetuar uma mudança de reação. A Força de Excitação (FE), que está relacionada à habilidade do indivíduo de resistir a uma estimulação intensa e prolongada sem manifestar inibição, revelou-se como uma característica menos presente neste grupo.

De modo geral esta caracterização do temperamento dos adolescentes ( $\mathrm{MO}>\mathrm{FI}>\mathrm{FE}$ ) permaneceu constante. Pequenas inversões entre as médias de MO e FI podem ser constatadas em adolescentes do sexo feminino e em participantes com 17 anos de idade, os quais apresentaram uma média ligeiramente maior no fator FI, ao invés do fator
MO. Comparando estes resultados aos dados obtidos por meio da PTS em avaliações de adolescentes e adultos, (Riello, 1999; Strelau et al., 1999) constataram resultados similares aos obtidos pela maior parte da amostra de adolescentes pesquisados: médias maiores em MO, intermediárias em FI e menores em FE ( $\mathrm{MO}>\mathrm{FI}>\mathrm{FE})$.

A análise das características de temperamento, quando considerada a variável sexo, evidenciou que os adolescentes do sexo masculino apresentaram médias mais elevadas nos fatores MO e FE quando comparados as adolescentes do sexo feminino, as quais obtiveram média mais elevada em FI. Tais resultados sugerem que os adolescentes do sexo masculino, participantes desta pesquisa, são indivíduos caracterizados por apresentar maior facilidade em responder adequadamente às mudanças do ambiente e que resistem à estimulação intensa e prolongada sem manifestar inibição.

Os dados da amostra brasileira pesquisada por Guzzo et al. (1996) e os resultados obtidos nas pesquisas transculturais da PTS (Strelau et al., 1999) são similares: participantes do sexo masculino com médias maiores em MO, FE e FI. Este último fator obteve, neste estudo, maior média entre os adolescentes do sexo feminino, mas a diferença constatada neste fator não foi significativa. Comparando-se estes resultados com os da amostra alemã, constata-se que são similares: participantes do sexo masculino com médias maiores que os femininos. De modo geral, a maioria dos países envolvidos nos estudos transculturais da PTS apresentam este padrão de resultados (Strelau et al., 1999).

A caracterização do temperamento, considerando a idade dos adolescentes, evidenciou uma tendência entre os adolescentes acima de 16 anos de apresentarem escores mais elevados nas dimensões MO e FI e de adolescentes de 14 anos apresentarem escores mais elevados na dimensão FE.

De acordo com Strelau et al. (1999), variações dos escores nas dimensões avaliadas pela PTS são esperadas; existe uma tendência a que as pontuações de FE e MO diminuam com o passar dos anos e que as pontuações de FI aumentem.

No que diz respeito às normas obtidas, estas refletem a caracterização de temperamento predominante nesta amostra ( $\mathrm{MO}>\mathrm{FI}>\mathrm{FE})$, pois a porcentagem de adolescentes que obteve pontuações mínimas em MO e FI foi pequena quando comparada a FE, o qual obteve médias menores em todos os grupos considerados.

\section{Considerações finais}

Os resultados obtidos evidenciaram que os índices de consistência interna dos fatores da PTS para a amostra deste estudo foram menores que os da literatura, porém considerados estatisticamente satisfatórios. Este fato instiga a continuidade das pesquisas envolvendo sua utilização junto à população brasileira.

Como instrumento de investigação das características de temperamento, a PTS evidenciou que esta amostra de adolescentes apresentou como características predominantes de temperamento a Mobilidade e a Força de Inibição e baixa 
Força de Excitação. Esta conjugação dos fatores da PTS caracteriza os adolescentes como indivíduos com habilidade de responder tão adequadamente quanto necessário a contínuas mudanças do ambiente, com facilidade de interromper um determinado comportamento quando necessário, ou ainda efetuar uma mudança de reação e que tendem a apresentar dificuldade de resistir a uma estimulação intensa e prolongada.

No que se refere à normatização da PTS 14 a 18 anos, dados do presente trabalho servirão de parâmetros para futuras pesquisas e comparações para caracterização do temperamento.

De modo geral, os resultados obtidos neste estudo confirmam dados de pesquisas anteriores realizadas com a PTS e indicam que esta é uma escala com bons índices de consistência interna e adequada para caracterizar o temperamento dos indivíduos.

Para futuros estudos, seria importante ampliar a amostra, envolvendo também a participação de adultos, e adotar planejamentos que incluam outros instrumentos e construtos de investigação das características de personalidade. Com isso, os resultados poderiam proporcionar informações mais detalhadas sobre as características psicométricas da PTS e sobre as correlações de diferentes construtos com os fatores da PTS, além de enriquecer os dados sobre as características de temperamento de diferentes grupos de indivíduos.

\section{Agradecimentos}

Agradecimentos à participação de C. C. M. Pinho, C. F. C. Carvalho, E. F. Gums, M. C. P. Scoz, T. S. Messias, bolsistas do LAMP.

\section{Referências}

Anastasi, A. (1988). Psychological testing (6 $6^{\mathrm{a}}$ ed.). Nova York: Macmillan. Aron, A., \& Aron, E. N. (1999). Statistics for psychology ( $2^{\mathrm{a}}$ ed.). Upper Saddle River, New Jersey: Prentice-Hall.

Carey, W. B., \& McDevitt, S. C. (1994). Prevention and early intervention. Nova York: Brunner/Mazel.

Carey, W. B. (1989). Practical applications in pediatrics. In G. A. Kohnstamm, J. E. Bates, \& M. K. Rothbart (Orgs.), Temperament in childhood (pp. 405-419). Chilchester: John Wiley and Sons.

Chess, S., \& Thomas, A. (1986). Temperament in clinical practice. New Jersey: Guilford Press.

Chess, S., \& Thomas, A. (1989). Issues in the clinical application of temperament. In G. A. Kohnstamm, J. E. Bates, \& M. K. Rothbart (Orgs.), Temperament in childhood (pp. 377-386). Chilchester: John Wiley and Sons.

Goldsmith, H. H., Buss, A. H., Plomin, R., Rothbart, M. K., Chess, S., Thomas, A., Hinde, R. A., \& McCall, R. B. (1987). What is temperament? Four approaches. Child Development, 58, 505-529.

Goldsmith, H. H., \& Rieser-Danner, L. A. (1986). Variation among temperament theories and validation studies of temperament assessment. In G. A. Kohnstamm (Org.), Temperament discussed - temperament and development in infancy and childhood (pp. 1-10). Bristol, Pennsylvania: Swets \& Zeitlinger Publishers.
Guzzo, R. S. L., Pereira, P. C., \& Valli, C. M. M. (submetido a publicação). Temperamento: análise de resumos publicados entre 1991 e 1997. Manuscrito submetido a publicação.

Guzzo, R. S. L., Riello, I. C., \& Primi, R. (1996). Construção de uma escala de temperamento para a realidade brasileira: um estudo baseado na PTS. Psicologia Escolar e Educacional, 1(1), 53-59.

Hofstee, W. K. B., \& De Raad, B. (1992). Integration of the Big Five and circumplex approaches to trait structure. Journal of Personality and Social Psychology, 63(1), 146-163.

Hoogendorp, N. (1992). Temperament bei kindern: die entwicklung und überprüfung einer kinderform des PTS (Temperamento de crianças: o desenvolvimento e avaliação de uma versão infantil da PTS). Diplomarbeit im Fakultät für Psychologie und Sportwissenschaft an Der Universität Bielefeld.

Ito, P. C. P., \& Guzzo, R. S. L. (2002). Diferenças individuais: temperamento e personalidade; importância da teoria. Estudos de Psicologia(Campinas), 19(1), 91-100.

Krathwohl, D. R. (1993). Methods of educational and social science research. Nova York: Longman.

Nunnaly, J. (1967). Psychometric theory. Nova York: McGraw Hill.

Pasquali, L. (1997). Psicometria: teoria e aplicações. Brasilia: Editora UnB.

Riello, I. C. (1992). Temperamento de adolescentes: estudo de um instrumento de avaliação. Dissertação de Mestrado não-publicada, Pontifícia Universidade Católica de Campinas, Campinas.

Riello, I. C. (1999). Temperamento: perfil de adolescentes com diferentes competências em natação. Tese de Doutorado não-publicada, Pontifícia Universidade Católica de Campinas, Campinas.

Strelau, J. (1991). Temperament and giftedness in children and adolescents. Comunicação apresentada em Nineth World Conference on Gifted and Talented Children, Hage, Holanda.

Strelau, J. (1998). Temperament: a psychological perspective. Nova York: Plenum Press.

Strelau, J., Angleitner, A., \& Newberry, B. H. (1999). The Pavlovian Temperament Survey (PTS): an international handbook. Seattle: Hogrefe $\&$ Huber Publishers.

Teglasi, H. (1995). Assessment of temperament. Eric Digest [on line], obtido em http://www.ed.gov/databases/eric-digest/ed389963.html.

Teglasi, H. (1998). Introduction to the mini-series: implications of temperament for the practice of School Psychology. School Psychology Review, 24(4), 475-478. 
1. A PTS foi desenvolvida a partir dos itens constituintes do Strelau Temperament Inventory - STI, construído por Strelau em 1969 e utilizado até o início da década de 1990, quando teve seus itens revistos e reformulados, recebendo o nome de Pavlovian Temperament Survey - PTS (Strelau et al., 1999).

Raquel Souza Lobo Guzzo, Doutora em Psicologia Escolar e do Desenvolvimento Humano, pela Universidade de São Paulo, é Professora Titular na Pontifícia Universidade Católica de Campinas.

Ricardo Primi, Doutor em Psicologia Escolar e do Desenvolvimento Humano, pela Universidade de São Paulo, é Professor na Universidade São Francisco.

Patrícia do Carmo Pereira Ito é doutoranda em Psicologia na Pontifícia Universidade Católica de Campinas e bolsista da FAPESP.

Endereço p/correspondência: (RSLG) Rua Santa Mônica, 136; Campinas, SP; CEP 13094-531. Tel.: (19) 32514206. Fax: (19) 3255-1970. E-mail: rguzzo@mpc.com.br 